\title{
Extensor Carpi Radialis Longus
}

National Cancer Institute

\section{Source}

National Cancer Institute. Extensor Carpi Radialis Longus. NCI Thesaurus. Code C52913.

A muscle originating at the lateral supracondylar ridge of the humerus and inserted at the back base of the second metacarpal bone that controls the radial extension and deviation of the wrist. 